\title{
POWER SYSTEM FOR THE EU:CROPIS SATELLITE - RESULTS FROM DESIGN TRADE-OFFS, ANALYSIS, SIMULATION AND TESTING
}

\author{
Jakob Fromm Pedersen \\ German Aerospace Center, Robert-Hooke-Str 7, 28359 Bremen, Germany, Email: jakob.pedersen@dlr.de
}

\begin{abstract}
The Eu:CROPIS satellite is the first satellite to be launched as part of the DLR Compact Satellite Program. The mission is currently in Phase D and is scheduled to be launched in 2017. The Eu:CROPIS satellite is a small satellite of about $1 \mathrm{~m}^{3}$, and a mass of $250 \mathrm{~kg}$. The nominal power consumption in science mode is approximately $200 \mathrm{~W}$ from which $\sim 50 \%$ is provided to the payloads. The design of the Eu:CROPIS power system design is driven by the primary payload which is a biological payload that has a very narrow temperature range, and the attitude control system which utilizes only magnetic torquers as actuators. The power system has been optimized in order to provide as much power as possible during the Launch and Early Operations Phase (LEOP) of the mission resulting in a design which for the nominal part of the mission is over-dimensioned.

This paper captures the trade-offs affecting the power system carried out on system level, which were made in the design phase of the mission, and will explain in more detail the analysis, simulation and testing related to the LEOP of the mission. Based on the simulations and resulting design iterations performed for the LEOP the probability of power balance in this phase has been increased significantly compared to the power system which was optimized for the nominal mission.
\end{abstract}

\section{INTRODUCTION}

The German Aerospace Center (Deutsches Zentrum für Luft- und Raumfahrt, DLR) is conducting research in practically all areas of satellite design. In the Institute of Space Systems, Department of Avionics Systems (RY-AVS) the focus is on the avionics suite; Onboard Data Handling, Communication and Power System.

In the domain of the Electrical Power System (EPS) the majority of resources in the last years have been allocated to supporting the Compact Satellite Program. The Compact Satellite Program is taken on by DLR in order to provide a platform for experiments and research in space. While the Compact Satellite Program strives to provide a standard platform, each mission is tailored specifically to the selected payloads. Since the program focuses on scientific payloads the power requirements and configuration (e.g. available area for solar panels, or the attitude profile) of the satellite will be different for each mission.

In providing support for the Compact Satellite Program, a design methodology has been developed, which allows to quickly assess the requirements for the power system based on requirements for the mission, as well as deriving requirements for the individual units which form the power system. Furthermore a test and verification setup has been established, allowing to simulate the various mission phases. For the Eu:CROPIS mission all power system units have been procured within Europe from established vendors of power system components.

In order to assess and evaluate the performance of the power system throughout all mission phases, the procured units are initially modeled in Matlab Simulink based on the data available from the supplier data sheets, and the mission phases are simulated in order to ensure that the power system is performing as assessed in the initial phases. Later the simulation model is updated based on more detailed values received from the suppliers and, if needed, the model is extended with data measured during unit testing.

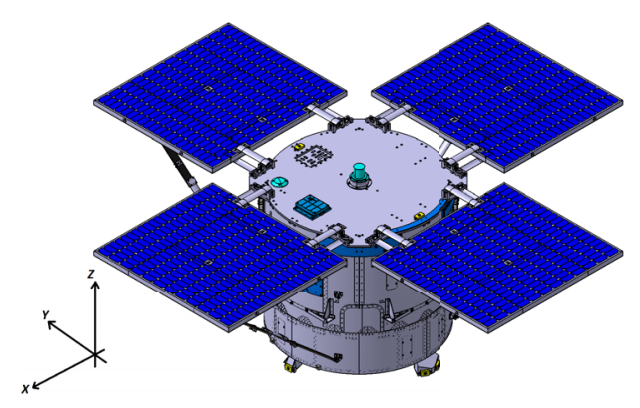

Figure 1. Eu:CROPIS Satellite in deployed configuration 


\section{EU:CROPIS MISSION AND DESIGN TRADE- OFFS}

The first mission from the DLR Compact Satellite program to be launched is the Eu:CROPIS mission, which will be launched in 2017. The Eu:CROPIS mission consists of one satellite of approximately $250 \mathrm{~kg}$, and dimensions of $1 \times 1 \times 1.2 \mathrm{~m}^{3}$. The primary payload for the Eu:CROPIS mission is a biological experiment which will assess the impact of various levels of gravity on a biological regenerative life support system[1]. The Eu:CROPIS satellite in the deployed configuration can be seen in Figure 1. The final configuration of the Eu:CROPIS satellite consists of a 'barrel' which contains all bus components and payloads, and four deployable solar panels. The payloads which are sensitive to varying gravity are located close to the edge of the 'barrel', and 'artificial gravity' is generated by spinning the Eu:CROPIS satellite around the Z-axis (see Figure 1).

Since the design of the Eu:CROPIS has undergone several iterations, the major trade-offs, design decisions and constraints for the power system will be explained in the following sections.

\subsection{Satellite Configuration}

One major design driver for the Eu:CROPIS satellite was the ability to be launched as a piggy-back satellite. In order to stay compatible with most launch opportunities the satellite, and thus also the power system, has been designed for low earth orbits between 550 and $650 \mathrm{~km}$. For the power system this leads to the worst case assumption of an sun synchronous orbit with an LTAN of 1200 , which results in an orbit of which approximately $38 \%$ is in eclipse.

In addition to the orbital constraints, the dimensions and mass of the satellite is limited by the availability of piggyback launches. In the early phases of the project a market survey was performed and the maximum envelope of $1 \times 1 \times 1.2 \mathrm{~m}^{3}$ was defined with a maximum mass of $250 \mathrm{~kg}$. A piggy-back launch opportunity is dependant on the available separation systems supported by the launch vehicle. Most opportunities similar in size and mass of the Eu:CROPIS envelope utilize a miniature separation ring, comparable to the separation rings used for bigger payloads. Since the launch profile is determined by the primary payload, no specific attitude can be ensured at the moment of separation. Due to the miniaturization of the separation rings and the comparably low mass of the satellite, the satellite will be separated with a non-zero angular momentum, resulting in a tumble. For Eu:CROPIS a maximum value of $10^{\circ} / \mathrm{s}$ was fixed during the early design phase. This value was later reduced to $5^{\circ} / \mathrm{s}$.

Due to the uncertainties in attitude and angular rates after separation, coupled with the limited actuation capability of the attitude control system, it was a requirement to have solar cells on minimum 4 faces of the satellite in order to ensure a minimal power generation. Based on the design of spin-stabilized satellites in the past, body

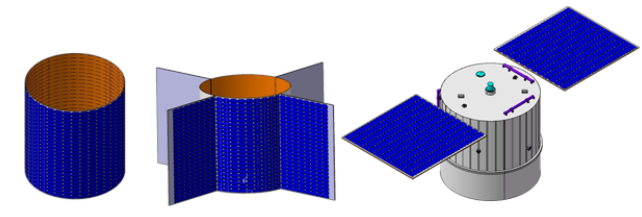

Figure 2. Early design considerations for the solar panels

mounted panels on the 'barrel' were investigated (see Figure 2).

Due to thermal requirements from the payloads and a rising power demand the concept was changed to deployable solar panels already in Phase 0. Based on the power requirements in Phase 0 and A, only two solar panels were required in order to ensure a positive energy balance, however in order to ensure that a minimum of four faces of the satellite was able to generate power, the required solar array area was split to four panels, which are stowed during launch (see Figure 3).

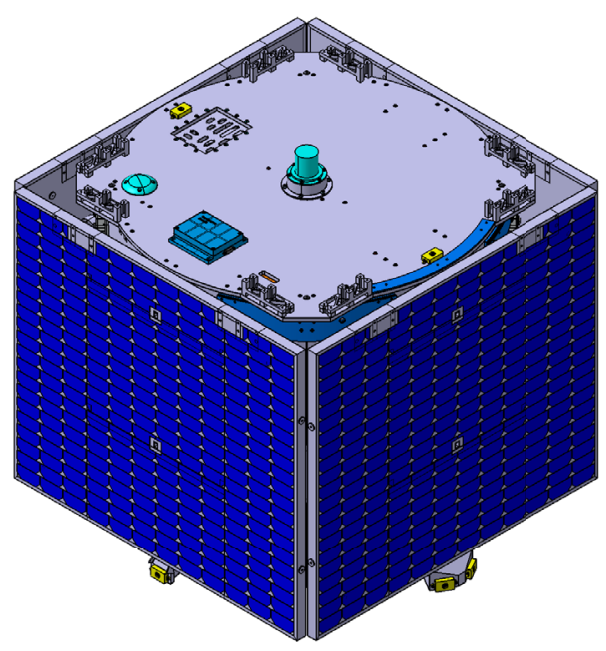

Figure 3. Eu:CROPIS Satellite in stowed configuration

\subsection{Power System Architecture}

When deciding on a power system architecture for a satellite in low earth orbit, the main decision is whether to use maximum power point tracking (MPPT) for the solar panel conversion, or to use direct energy transfer. The decision depends on the mission profile on the operating conditions for the solar panels, and is a trade-off between between simplicity, efficiency and cost [2][3][4][5]. Since the Eu:CROPIS satellite is relying on the power generation of the solar panels both in stowed and deployed configuration, for which the operating temperature of the solar panels is significantly different, a power regulator implementing MPPT was chosen. Following the trend of small satellites comparable to Eu:CROPIS in power demand and size, an unregulated 
(or battery-regulated) power bus with a bus voltage of 22-34V was chosen as the back-bone of the power system. The unregulated power bus simplifies the design of the power system by discarding the battery charge and discharge regulator, however increases the input voltage range for the satellite units compared to a regulated bus. Due to the choice of using an MPPT regulator the maximum power available from the solar panels can be utilized independent of the battery voltage. The Eu:CROPIS Power system architecture can be seen in Figure 4.

As a result from a trade-off between redundancy, mass, volume and cost the PCDU was procured with only two Array Power Regulators (APRs). The APRs are not coupled in parallel, and search the maximum power point for each input independently. When the solar panels are deployed, all panels will be sun-pointing (see Figure 1). Since all four panels are identical, the temperature and thus the panel I-V profile will be identical for all panels. However during the Launch and Early Operations Phase (LEOP) of the mission, Eu:CROPIS is required to generate power in the stowed configuration (see Figure 3 ). In order to ensure that each APR only sees one panel generating power at any given time, two opposite panels are connected in parallel to one APR. This prevents the overlapping of two different I-V curves, which could trick the MPPT into locking on the wrong maximum power point. Additionally this design allows to reduce the required maximum power handling capability for each APR.

In order to simplify the power system design, it was decided at an early point to only distribute the main power bus to the satellite units. All units which are not compatible with the main bus voltage (e.g. $5 \mathrm{~V}$ inputs), are required to implement DC/DC converters prior to the unit input. Since most units with a $5 \mathrm{~V}$ input do not provide galvanic isolation between the primary and secondary power, the galvanic isolation is provided by the DC/DC converter. The drawback of this implementation is an increased power consumption, in particular for units with low input current. Due to the galvanic isolation in most cases the DC/DC dissipates as much power as the unit powered. For future DLR compact satellite missions, a more detailed trade-off will be made regarding this decision, in particular if the power budget is narrow.

\subsection{Launch and Early Operational Phase (LEOP)}

Seen from the power system, the LEOP is the most critical part of the Eu:CROPIS mission. Due to the narrow temperature requirements of the primary payload, the approach of defining a satellite mode, where all non-essential units are powered off cannot be utilized on Eu:CROPIS. If the temperature of the primary payload gets too low, the biological cells and organisms contained in the payload will start to die, which will lead to mission

\begin{tabular}{l|c} 
Satellite Mode & Power Consumption \\
\hline Low Power & $36 \mathrm{~W}$ \\
ACS Mode & $72 \mathrm{~W}$ \\
Safe Mode & $102 \mathrm{~W}$ \\
Science Mode & $190 \mathrm{~W}$
\end{tabular}

Table 1. Eu:CROPIS Satellite Modes and Power Consumption

failure. In order to keep the biology within the required temperature range heaters are mounted to the critical areas of the payload.

The attitude control system for the Eu:CROPIS satellite consists of various sensors for determining the attitude and relies solely on magnetic torquers for control [6]. Since the size and angle of the angular torque which can be generated varies with the Earth magnetic field, initial worst case analysis showed that approximately 4-5 hours were required in order to achieve a sun-pointing attitude with the $+\mathrm{Z}$ axis.

During Phase 0 - B the power concept for the LEOP was to rely completely on the spacecraft battery as a power source for worst case analysis, and the requirement specification for the battery was based on this assumption. Due to power creep of satellite units early in the detailed design phase it was decided to perform a larger design iteration on the satellite modes to re-assess the solar panel sizing and redefine the autonomy of the satellite during the LEOP. This exercise forced all subsystems to rethink the power demand during the LEOP and reduce the power demand to "need to have" rather than "nice to have". As an outcome of the design iteration, the modes of the satellite were re-defined (see Table 1).

The Eu:CROPIS satellite automatically enters the "Low Power" mode, where only the Communication Receivers and Onboard Datahandling is active, upon detecting the separation from the launcher. If the battery state of charge is 'good', the satellite will change to "ACS" mode, where the attitude control system is active, and the satellite will attempt to achieve sun-pointing attitude with the stowed solar arrays. If at any point the temperature in the payload is critically low, the payload heaters are activated. If the battery state of charge is detected as 'low', the satellite will switch to the "Low Power" mode, and wait for the battery to charge until the state of charge is 'good'. If the battery state of charge is detected as 'critical', the satellite will deactivate the payload heater loops regardless of the payload temperature. The autonomy of the Eu:CROPIS satellite is shown in Figure 5.

In addition to iterating the satellite modes and reassessing the autonomy of the satellite, the solar array sizing was re-analyzed. During Phase 0-B the design driver for the solar panels was the nominal part of the mission, where a solar array output of $\approx 380 \mathrm{~W}$ was required (equal to $95.53 \mathrm{~W}$ per panel). In order to increase the probability that a positive power balance can be maintained during the LEOP, a trade-off between cost, mass and probability of power balance, in case attitude control can not be obtained, was performed. Based on 


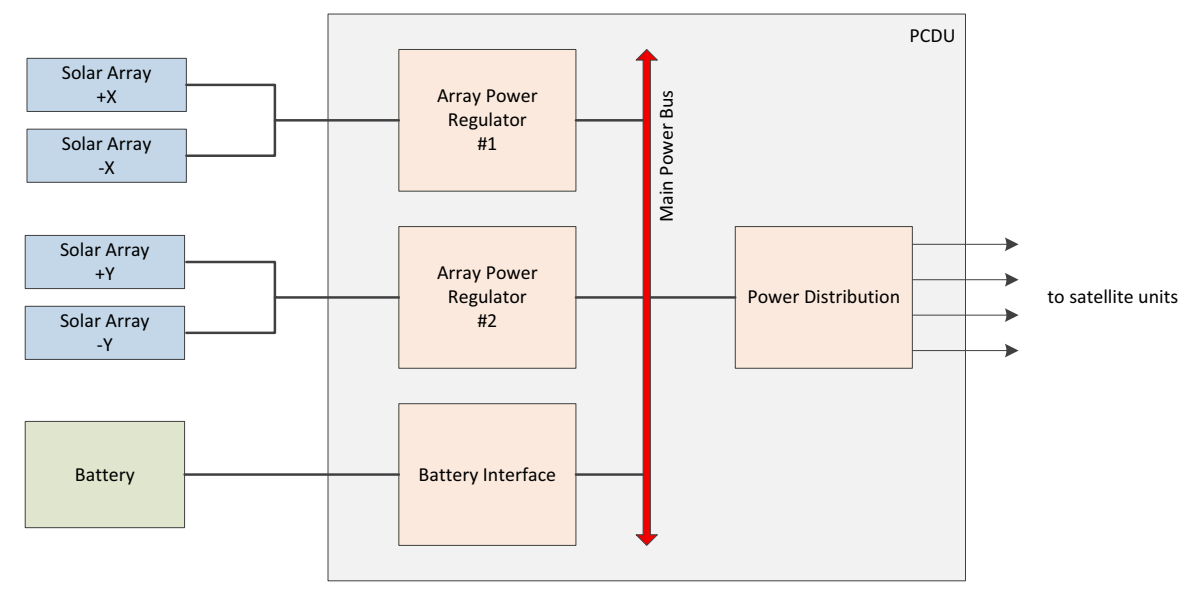

Figure 4. Eu:CROPIS Power System Architecture

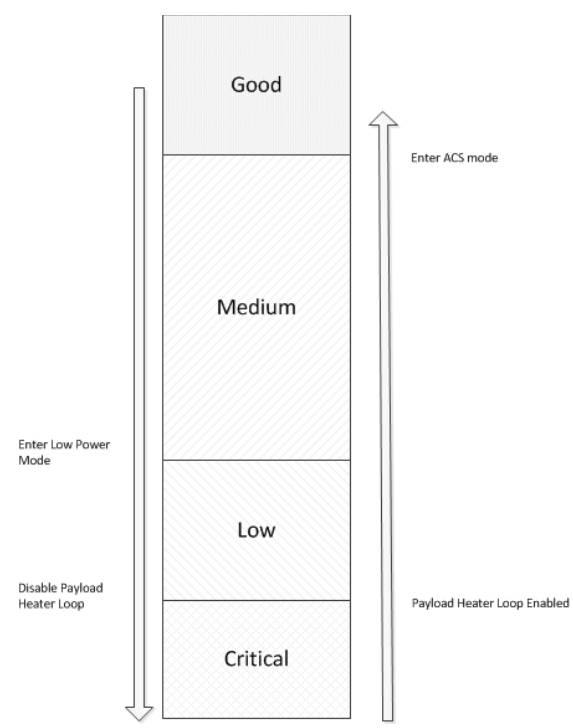

Figure 5. Power System State of Charge and Autonomy

the analysis it was decided that the panel output was to be maximized, resulting in a solar panel capability of $189 \mathrm{~W}$ worst case. The combined power capability of two solar panels is more than what a single APR can convert. However, due to the connection scheme of the solar panels to the APRs, a single APR will never see more than one illuminated solar panel during the LEOP phase.

\section{POWER SIMULATION AND ANALYSIS}

In order to analyse the power generation and power balance during the LEOP a power system model has been constructed in Matlab Simulink. The model used for the probability analysis includes the kelly cosine relation between solar panel incidence angle and power output [7], and a minimum power deadband for the APR. For the simulation model fixed efficiency parameters are assumed for the PCDU, Battery and Solar Array. The assumptions for the model are either based on worst case values provided by the supplier, or based on tests performed on the delivered units.

In order to assess the probability of a power balance in case of a malfunction onboard the satellite during the LEOP, an analysis of the power generation during a random tumble has been performed. An amount of 10.000 'tumble cases' have been constructed for the satellite, where it is assumed that the satellite is rotating around a fixed random vector. In addition, the location of the sun vector is randomized. The power output for a single revolution has been simulated using the Simulink model. Under the assumption that the satellite has a minimum angular rate of $0.25^{\circ} / s$ (equal to approximately 4 revolutions per orbit), the power during one revolution has been assumed as the average power output during the entire orbit, excluding the eclipse period.

Based on the power generation output, the available power for the satellite has been calculated. A histogram of the results can be seen in Figure 6, and the cumulative distribution function can be seen in Figure 7. Based on the cumulative distribution function, the probability of positive power balance has been calculated for "Low Power" and "ACS" mode respectively, with varying payload heater power. The results can be seen in Table 2 .

\begin{tabular}{l|c|c|c} 
Satellite & Heater & \multicolumn{2}{|c}{ Panel Power } \\
Mode & Power & $189.00 \mathrm{~W}$ & $95.53 \mathrm{~W}$ \\
\hline Low Power & 0 & $99.60 \%$ & $95.32 \%$ \\
Low Power & $10 \mathrm{~W}$ & $99.17 \%$ & $64.04 \%$ \\
Low Power & $20 \mathrm{~W}$ & $98.53 \%$ & $16.12 \%$ \\
\hline ACS & 0 & $95.19 \%$ & $0.01 \%$ \\
ACS & $10 \mathrm{~W}$ & $87.35 \%$ & $0.00 \%$ \\
ACS & $20 \mathrm{~W}$ & $60.78 \%$ & $0.00 \%$
\end{tabular}

Table 2. Probability For Positive Power Balance

As can be seen in the table, the probability of power balance in all cases is increased significantly with the fully 


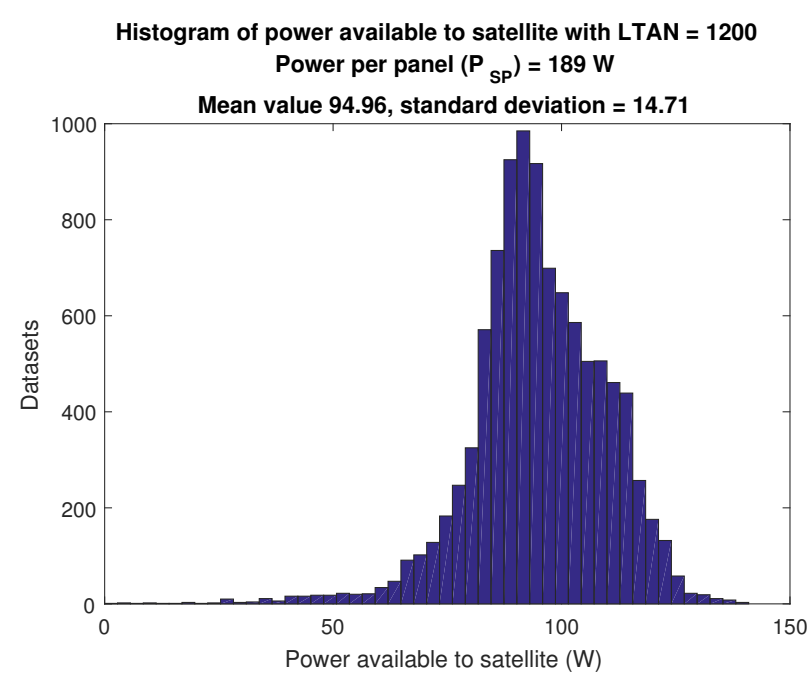

Figure 6. Histogram of simulation results with panel power of $189 \mathrm{~W}$

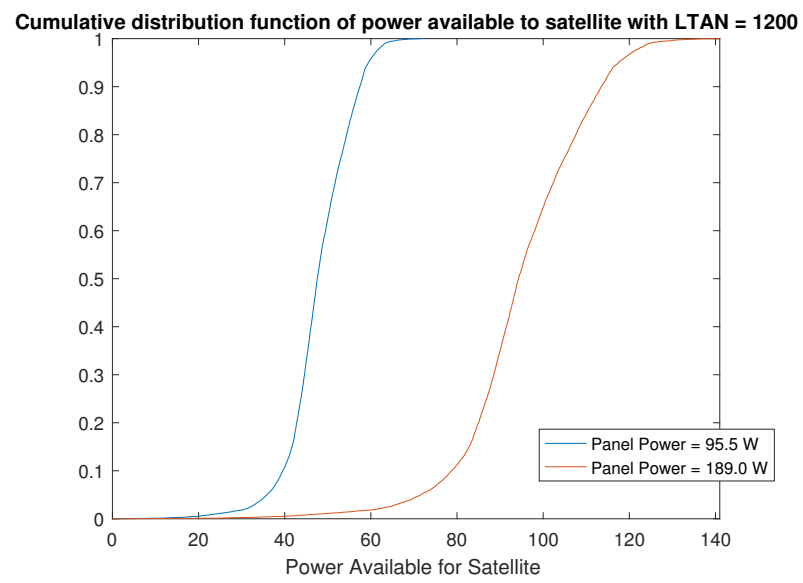

Figure 7. Cumulative distribution function of simulation results

utilized solar panel (189W per panel), compared to the solar panels designed based on power demand during the nominal mission (95.53 W per panel).

\section{POWER SYSTEM TESTING}

In order to verify that the Eu:CROPIS power system is performing within specifications and according to the design, several tests have been performed on both unit and system level. For the power system a strong reliance on unit testing by the suppliers has been chosen, due to limited resources and facilities available to the project. Instead the focus has been on the system tests, both on power system and satellite level.

On satellite level the interaction between the power system and the onboard data handling system has been verified, by testing the system behavior in various test-cases. The failure handing has been verified by inducing a short-circuit on an LCL output. Based on the configuration of the given LCL it is verified, by telemetry and by measuring the PCDU output, that the system reacts as commanded, e.g. that the output is re-activated by software, or that the satellite automatically enters safe mode. Also the mode-transitioning has been verified by varying the battery voltage using a battery simulator. It has been verified that the satellite is changing the mode autonomously according to the battery state of charge, which is determined based on the battery voltage (refer to section 2.3).

The Eu:CROPIS Engineering Model is not a full representation of the flight model, e.g. most redundant units are not included in the EM. In addition to the missing units, several units utilize COTS components in order to reduce cost and lead time, which results in a different power consumption than for the FM units. As a consequence it has not been possible to verify the satellite power budget and the energy balance during the nominal mission. Due to the large power margin for the nominal mission phase, this is not seen as critical. Rather than the nominal mission phase the majority of the testing performed related to the power system has been focused on the LEOP of the mission. Two extensive test campaigns have been performed related to the LEOP; 'MPPT Efficiency' and 'Verification of Tumble Case', and both will be described in the following sections.

\subsection{MPPT Efficiency}

In order to maintain a stable attitude, the Eu:CROPIS satellite is required to spin during all mission modes [6]. During the LEOP Mode, the attitude control system will maintain a spin rate of 5 revolutions per minute (RPM) in a barbeque (BBQ) mode around then Z-axis, with the spin axis being perpendicular to the sun, and thereby optimizing the power output. During the nominal mission, the satellite will have a spin rate of 20 or 30 RPM, depending on the experiment phase.

During the LEOP mode the solar panels are stowed. Due to the BBQ mode of the satellite, it is assumed that the panel temperature is relatively constant and therefore it can be assumed that the $V_{o c}$ and $V_{m p}$ parameters of the solar panels are constant during the LEOP phase. However due to the rotation of the satellite, the panel current will vary according to the kelly cosine function of the incidence angle. The satellite is required to rotate with a minimum of approximately 2 RPM in order to remain stabilized during the BBQ mode, however a spin rate of 5RPM is preferred in order to provide a higher margin against external disturbances. A spin rate of 5 RPM, corresponds to an angular velocity of 30 degrees per second, which is also the angular rate of the solar incidence angle on the solar panels. Due to the relative large angular rate of the incidence angle, the current parameters of the solar panel will change with a high slew rate, in particular when the panels are coming 'into sunlight'. In order to verify that the MPPT can follow the change in panel parameters, while still maintaining 

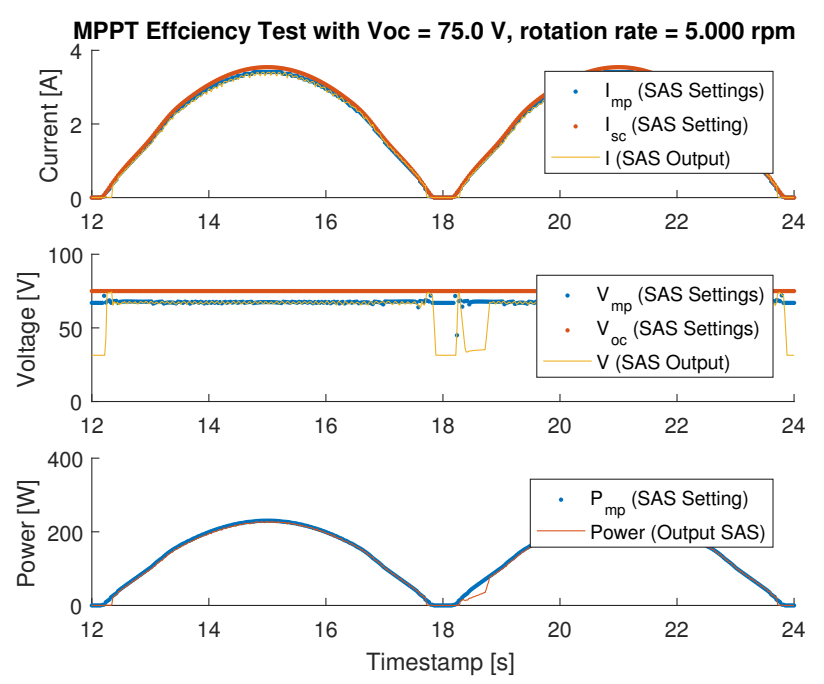

Figure 8. $V_{o c}=75 \mathrm{~V}, 5 \mathrm{RPM}, P_{m p} 230 \mathrm{~W}$

the MPP, a series of tests has been performed on the Eu:CROPIS PCDU EM, in order to verify the MPPT efficiency for a rotating satellite.

The MPPT efficiency has been measured for panel parameters with $V_{o c}$ between $45-75 \mathrm{~V}$, which is the expected range of the Eu:CROPIS solar arrays, and a $P_{m p}$ of $115 \mathrm{~W}$ and $230 \mathrm{~W}$. The rotational rate was varied between 0.5 and 15 revolutions per minute.

All MPPT efficiency tests have been performed using the Keysight E4360 Solar Array Simulator (SAS), which allows to generate a list of I-V profiles with a minimum dwell time of $30 \mathrm{~ms}$. Assuming a fixed $V_{o c}$ and $V_{m p}$ the $I_{m p}$ and $I_{s c}$ parameters have been calculated and transferred to the SAS using the Matlab Instrument Control Toolbox. In order to determine the efficiency of the MPPT the current and voltage on the output of the SAS is logged during 10 rotations. The MPPT efficiency has been determined based on the power being drawn from the SAS, compared to the power available according to the SAS settings during 8 rotations (the first and last rotation are not being used for the efficiency calculations).

In Fig. 8 the measurements from a single rotation can be seen. During the illumination of the first solar panel, the PCDU is tracking the power point during the entire half-sine, however during the illumination of the second panel, the MPP is not tracked during the initial part of the half-sine. Once the MPP tracking has been achieved, the MPP is tracked for the remaining part of the panel illumination.

Due to the technical limitations in general for a SAS in terms of accurately simulating a solar panel [8], and the minimum dwell time between two adjacent sampling points, the efficiency measurements are subject to uncertainties. Due to the dwell time limitations the solar panel parameters are varying with an frequency of $33.3 \mathrm{~Hz}$, while the PCDU is tracking the MPP with a higher frequency. This allows the PCDU to find the MPP and 'stabilize' before the solar panel parameters

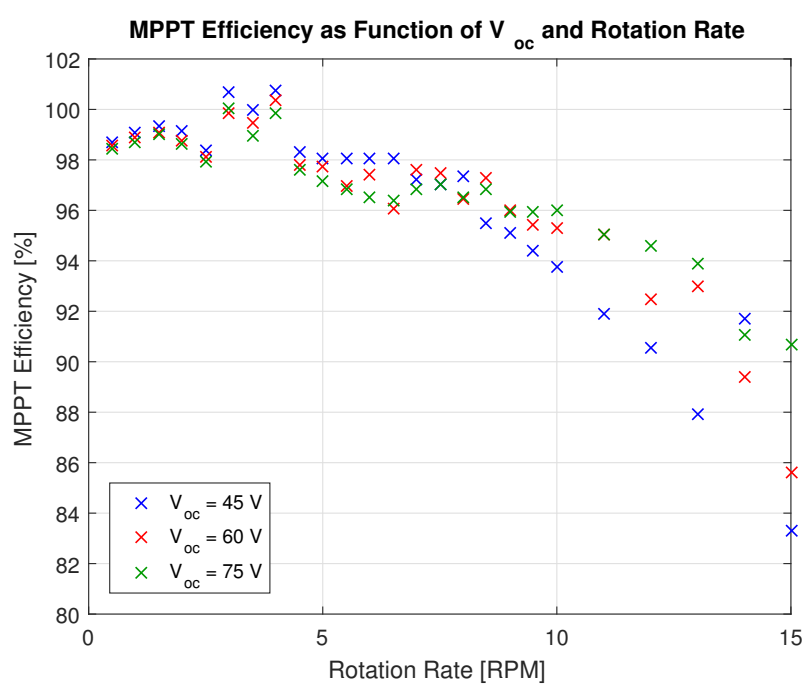

Figure 9. MPPT Efficiency as function of $V_{o c}$ and Rotation Rate with $P_{m p}=230 \mathrm{~W}$

are changed, whereas the solar panel parameters on orbit will change as a continues function when the satellite is rotating. The SAS output voltage and current is sampled with the maximum frequency of $50 \mathrm{~Hz}$, so it is not possible to identify the MPP tracking, and inspect how the PCDU reacts to a sudden change in solar panel parameters. It is however observed that once the PCDU has found the MPP during the illuminated phase, the MPP is maintained. It is estimated that the uncertainty for the efficiency measurements is within $\pm 2 \%$, based on the measured values, and the user guide for the SAS [9]. This uncertainty also accounts for measurements with an efficiency higher than $100 \%$. The results from the efficiency tests can be seen in Fig. 9 and Fig. 10 for $P_{m p}=230 \mathrm{~W}$ and $P_{m p}=115 \mathrm{~W}$ respectively. As can be seen in the figures, the efficiency is constant until a rotational rate of approximately of 5 RPM, after which the efficiency begins to decline. Based on the measurements, no correlation between the $V_{o c}$ parameter and the efficiency can be found. Only a small decline in MPPT efficiency was seen with a $P_{m p}=115 \mathrm{~W}$ compared to $P_{m p}=230 \mathrm{~W}$, however it can not be excluded that the difference is due to measurement inaccuracies. In addition to the results shown in Fig. 9 and Fig. 10 measurements were performed up until 30 RPM, in order to asses whether the primary mission could be performed with stowed solar panels, however the results were inconclusive, and a large variation in the efficiency was seen between the various runs.

Based on the measurements performed it is concluded that the efficiency of the MPPT is sufficient to allow the Eu:CROPIS satellite to rotate with 5 RPM during the LEOP phase.

In addition to verifying the MPPT efficiency for the rotating satellite, the efficiency was also measured for a solar panel with fixed parameters. This test confirmed that the MPPT efficiency is $>99 \%$. 


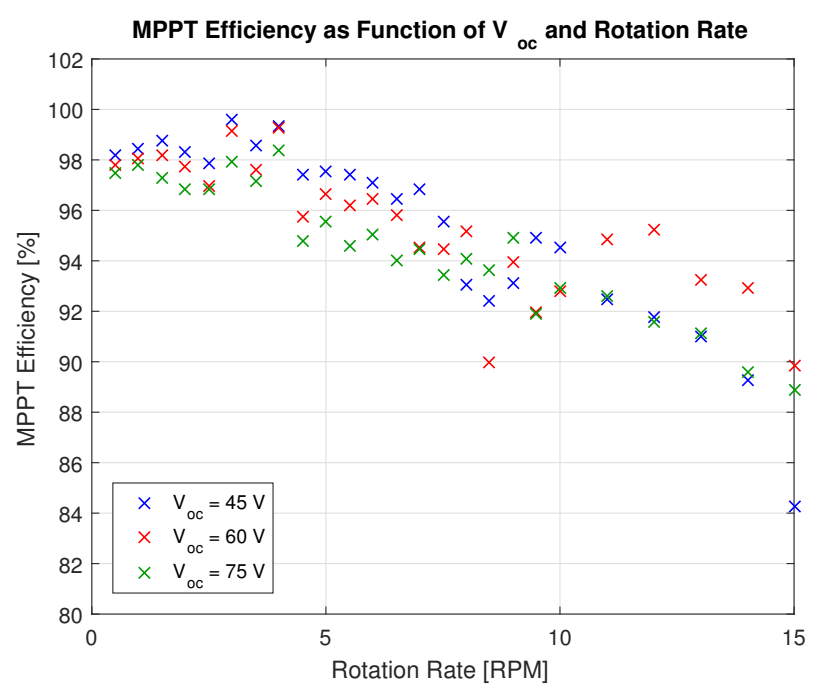

Figure 10. MPPT Efficiency as function of $V_{o c}$ and Rotation Rate with $P_{m p}=115 \mathrm{~W}$

\subsection{Verification of Power Generation during ran- dom Tumble}

As explained in section 3 an extensive simulation and analysis has been performed regarding the probability of power balance in the case that no attitude control is achievable. In order to verify the assumptions used for the analysis, a test campaign has been performed to determine the correlation between the simulation and the measured values.

For the simulation and test the solar array temperature has been assumed to be $50^{\circ} \mathrm{C}$, which is the expected maximum temperature for the solar panels during the LEOP BBQ mode, and the satellite is assumed to be rotating with a rate of 5 RPM. The MPPT efficiency has been assumed to be $98.0 \%$ based on the results from the MPPT efficiency measurements for a rotating satellite, explained in the previous section. A total of 4.000 tumble cases have been simulated using the same SAS as used for the MPPT efficiency measurements. In Fig. 11 the cumulative distribution function can be seen for the SAS power output, the SAS power settings and the SAS power setting corrected with the MPPT efficiency parameter. As can be seen in the figure the SAS settings corrected for MPPT efficiency correlate very well with the SAS output values, and it is therefore concluded that the MPPT efficiency assumption is valid for the random tumble simulation.

\section{CONCLUSION}

The Eu:CROPIS power system, like any other subsystem on-board the satellite, has undergone several minor and major changes throughout the design phases. Many changes were triggered due to changes in power demand from the satellite subsystems. However, the biggest trade-offs, some of which are explained in this paper,

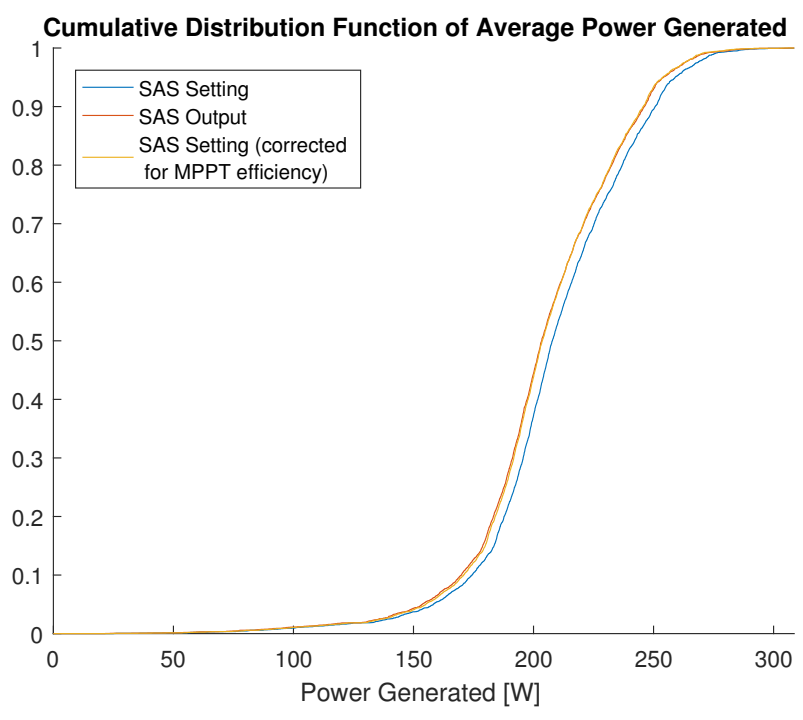

Figure 11. Cumulative distribution function of 4000 random tumble cases

were due to risk mitigation or requirements imposed due to external factors. The largest design driver in the later design phases of the Eu:CROPIS mission has been the LEOP, and several changes have been carried out due to the requirements and assumptions for this phase.

The Eu:CROPIS satellite has a power system which is over-dimensioned for the nominal science phase, however the robustness against failures in the early phases of the mission has been increased significantly. The increase in robustness has been achieved by applying good engineering when re-assessing the spacecraft modes and autonomy, and by increasing the performance of the solar array to the maximum extent possible within the boundaries of the satellite envelope.

During the Eu:CROPIS development phase, the power system has been designed, simulated and tested. A simulation framework has been established in Matlab Simulink, and is continuously expanded to include more parameters and reflect test results obtained by tests on the real hardware.

A significant effort has been invested in testing the power system, in particular for the solar array input. Utilizing solar array simulators, the solar array of a spinning satellite has been simulated with rotational rates of up to 15 RPM, as well as a significant number of random tumble cases. Based on the tests performed, full confidence has been gained that the power system for the Eu:CROPIS mission will perform according to the expectations.

\subsection{Future Work}

Based on the Eu:CROPIS project several topics have been identified which need further analysis in order to improve the accuracy of the simulation and testing. In addition several tasks which must be performed in order to sup- 
port the flight phase of the Eu:CROPIS mission are still open.

As explained in section 4.1, the MPPT efficiency measurements for a rotating satellite are associated with uncertainties due to the dwell time limitations in the solar array simulator. While the results obtained for the Eu:CROPIS mission provides confidence that the system will perform as expected, more detailed analysis and measurements will have to be performed in order to assess the impact on future missions, and to what extent the measurements are valid.

In order to model the behavior of the flight model the model of the power system will have to be updated to reflect the measured performance during the verification campaign. This includes updating the Matlab Simulink model to reflect dynamic efficiencies for the PCDU. The parameters for the model will initially be based on measurements performed by the supplier on the FM, and will later be updated with measurements performed on the Eu:CROPIS FM, if applicable.

Finally work must be invested in the preparation of the EGSE, in particular for the control software, allowing to perform mission simulations without the need of Matlab Simulink. The EGSE and associated software will be used to perform all functional tests on the Eu:CROPIS FM during the verification campaign on system level, and during the flight campaign. The EGSE must be able to simulate the solar panel input for the nominal and worst case orbits for the nominal mission phase, as well as for the LEOP. Once all tests on the Eu:CROPIS FM are completed, the EGSE will be used to operate the Eu:CROPIS ground reference model.

\section{REFERENCES}

[1] Jens Hauslage, Michael Lebert, and Hartmut Müller. Eu:CROPIS - euglena and combined regenerative organic-food production in space. In Life in Space for Life on Earth (Joint Life Sciences Meeting of ISGP, ESA and CSA), Juni 2014.

[2] W. Denzinger. Electrical power subsystem of globalstar. In 4th European Space Power Conference, September 1995.

[3] Antonio De Luca and Giovanni Chirulli. Solar array power conditioning for a spinning satellite. In $8 t h \mathrm{Eu}$ ropean Space Power Conference, September 2008.

[4] Toni Fabio Catalano, Stefano Costantini, and Giorgio Daprati. Sentinel-1 eps architecture and power conversion trade-off. In 9th European Space Power Conference, June 2011.

[5] Emanniel Colas. Electrical power subsystem of globalstar 2nd generation satellite. In 9th European Space Power Conference, June 2011.

[6] Ansgar Heidecker, Takahiro Kato, Olaf Maibaum, and Matthew Hölzel. Attitude control system of the Eu:CROPIS mission. In 65th International Astronautical Congress. International Astronautical Federation, Oktober 2014.
[7] Mukund R. Patel. Spacecraft Power System. CRC Press, 2005.

[8] A. Fernandez, C. Baur, and F. Gomez-Carpintero. Solar array hysteresis and its interaction with the mppt system. In Proceeedings of the European Space Power Conference 2014, April 2014.

[9] Keysight Technologies Series E4360 Modular Solar Array Simulator. 